OPEN ACCESS

Edited by:

Christe Weiss,

University of Heidelberg, Germany

Reviewed by:

Veronica Dusi,

University of Turin, Italy

Michael Behnes,

University of Heidelberg, Germany

*Correspondence:

Fa-Po Chung

marxtaij@gmail.com

Tzu-Ting Kuo

absintholic@hotmail.com

Specialty section:

This article was submitted to

Cardiac Rhythmology,

a section of the journal

Frontiers in Cardiovascular Medicine

Received: 06 March 2021 Accepted: 25 June 2021

Published: 15 July 2021

Citation:

Li G-Y, Chen $Y$-Y, Chien $K-L$, Lin $Y-J$, Kuo T-T and Chung F-P (2021) Clinical

Significance of Ventricular

Tachyarrhythmias in Patients

Undergoing Valve Replacement: A Nationwide Population-Based Study.

Front. Cardiovasc. Med. 8:676897.

doi: 10.3389/fcvm.2021.676897

\section{Clinical Significance of Ventricular Tachyarrhythmias in Patients Undergoing Valve Replacement: A Nationwide Population-Based Study}

\author{
Guan-Yi Li ${ }^{1}$, Yun-Yu Chen ${ }^{1,2}$, Kuo-Liong Chien ${ }^{2}$, Yenn-Jiang Lin ${ }^{1,3}$, Tzu-Ting Kuo ${ }^{4,5 *}$ and \\ Fa-Po Chung ${ }^{1,3 *}$ \\ ${ }^{1}$ Division of Cardiology, Department of Medicine, Taipei Veterans General Hospital, Taipei, Taiwan, ${ }^{2}$ Institute of Epidemiology \\ and Preventive Medicine College of Public Health, National Taiwan University, Taipei, Taiwan, ${ }^{3}$ Institute of Clinical Medicine \\ and Cardiovascular Research Center, National Yang-Ming University, Taipei, Taiwan, ${ }^{4}$ Division of Cardiovascular Surgery, \\ Department of Surgery, Taipei Veterans General Hospital, Taipei, Taiwan, ${ }^{5}$ Department of Medicine, School of Medicine, \\ National Yang Ming University, Taipei, Taiwan
}

Background: The clinical significance and outcomes of ventricular tachyarrhythmias (VTa) in patients undergoing valve replacement have rarely been reported.

Objective: This study aimed to investigate the incidence and outcome of VTa after surgical valve replacement.

Methods: We conducted a population-based retrospective cohort study using data obtained from the Taiwan National Health Insurance Research Database. In total, 10,212 patients were selected after 1:1 propensity-score matching based on the type of prosthetic valve used (mechanical vs. bioprosthetic). Various outcomes during long-term follow-up were analyzed.

Results: After a median follow-up period of 59.6 months, the crude incidence rate of VTa after surgical valve replacement was 4.8/1,000 person-years, and the cumulative incidence of VTa persistently increased after surgery. Furthermore, the occurrences of VTa after valve replacement significantly increased the risk of cardiovascular (CV) death $(P<0.001$, HR 1.67, 95\% Cl 1.41-1.96), stroke- $(P<0.001, \mathrm{HR} 1.66,95 \% \mathrm{Cl}$ 1.37-2.01), atrial fibrillation- $(P<0.001, \mathrm{HR} 2.80,95 \% \mathrm{Cl} 2.42-3.24)$, and congestive heart failure-related hospitalization $(P<0.001$, HR 2.61, 95\% Cl 2.30-2.95). Among patients with $\mathrm{VTa}$, all-cause mortality $(P=0.001, \mathrm{HR} 0.49,95 \% \mathrm{Cl} 0.32-0.75)$ and $\mathrm{CV}$ death $(P=0.047$, $\mathrm{HR} 0.58,95 \% \mathrm{Cl} 0.34-0.99)$ in those with implantable cardioverter-defibrillator (ICD) implantation were lower than those without.

Conclusion: The crude incidence rate of VTa after surgical valve replacement was 4.8/1,000 person-years, and the cumulative incidence of VTa persistently increased during follow-up. The presence of $\mathrm{VTa}$ after surgical valve replacement increases hospitalization and CV death, while ICD implantation reduced the mortality rate in these patients.

Keywords: bioprosthetic valve, implantable cardioverter-defibrillator, mechanical valve, valve replacement, ventricular tachyarrhythmia 


\section{INTRODUCTION}

Ventricular tachyarrhythmias (VTa), including ventricular tachycardia (VT) and ventricular fibrillation (VF), often contribute to worse clinical outcomes in patients with structural heart disease, such as valvular heart disease (VHD) (1). Of note, VHD might lead to structural remodeling, which may result in myocardial stretching and scar formation and consequently increase the probability of ventricular arrhythmogenesis (2). Despite surgical valve replacement, patients could still be at risk of sudden cardiac death due to arrhythmias, progressive myocardial dysfunction, and valve-related complications (3). This finding was supported by the fact that arrhythmias account for $6 \%$ of deaths in 1,533 patients who underwent aortic or mitral valve replacement (4). However, only a few studies focused on the incidence of VTa after valve replacement and associated clinical outcomes. This study aimed to assess the incidence of VTa after surgical valve replacement, the correlation between prosthetic valves and VTa, and the clinical impact of VTa on various outcomes, including mortality and hospitalization, using a nationwide population-based database. Furthermore, we investigated the role of implantable cardioverter-defibrillator (ICD) implantation in these patients. We believe that the abovementioned findings could improve the understanding of the clinical significance of VTa after valve replacement.

\section{MATERIALS AND METHODS}

\section{Study Design and Participants}

We conducted a population-based retrospective cohort study, and data were collected from January 1, 2000, to December 31, 2011, using the National Health Insurance Research Database (NHIRD). Patients receiving their first surgical valve replacement were grouped based on the procedural codes of the Specifications of the National Voluntary Consensus Standards for Cardiac Surgery: bioprosthetic valve replacement (procedure codes 35.21 , $35.23,35.25$, and 35.27$)$ and mechanical valve replacement (procedure codes 35.22, 35.24, 35.26, and 35.28). The exclusion criteria were as follows: patients aged $<20$ years, patients who underwent both bioprosthetic and mechanical valve replacement, and patients who presented with lethal ventricular arrhythmias or who underwent an ICD implantation before the procedures. Patients underwent an ICD implantation according to the reimbursement criteria of National Health Insurance (NHI), including prior sudden cardiac arrest due to $\mathrm{VTa}$, documented sustained VT/VF, or syncope caused by VA. ICD implantation was identified by the material codes of the NHI.

This study was approved in accordance with the Good Clinical Practice Guidelines by the Institutional Review Board of Taipei Veterans General Hospital.

\footnotetext{
Abbreviations: AF, atrial fibrillation; BBR, bundle branch reentry; CHF, congestive heart failure; CI, confidence interval; CV, cardiovascular; ESRD, end-stage renal disease; HR, hazard ratio; ICD, implantable cardioverter-defibrillator; LV, left ventricular; NHIRD, National Health Insurance Research Database; PSM, propensity-score matching; VF, ventricular fibrillation; VHD, valvular heart disease; VT, ventricular tachycardia; VTa, ventricular tachyarrhythmia.
}

\section{Databases and Characteristics of the Participants}

The Taiwan Collaboration Center of Health Information Application, Ministry of Health and Welfare, provided all datasets of the NHIRD. Taiwan's NHI program enrolled 26 million people (covering $99 \%$ of the country's population), including data on outpatient visits, hospital care, prescribed medications, and the National Death Registry. We obtained permission from the National Research Institute for the Department of Health and the Health Promotion Administration, Ministry of Health and Welfare.

The underlying diseases were identified according to the International Classification of Diseases, 9th Revision-Clinical Modification (ICD 9-CM) codes. To be coded for the study, the diagnosis must have been recorded twice in the outpatient records or at least once in the inpatient records. By linking to the NHIRD, we identified the following clinical variables: age, sex, type of valve replacement, number of valve replacements, history of diabetes mellitus, hypertension, chronic obstructive pulmonary disease, end-stage renal disease (ESRD) (5), congestive heart failure (CHF), coronary artery disease, and stroke.

\section{Outcomes During Long-Term Follow-Up}

The outcomes assessed in this study were all-cause mortality, cardiovascular (CV) death (ICD 9-CM codes 390-429), VTa(ICD 9-CM codes 427.1 and 427.4), CHF- (ICD 9-CM code 428), stroke- (ICD 9-CM codes 430-438), and atrial fibrillation (AF)related hospitalization (ICD 9-CM code 427.31). VTa was defined as ventricular tachycardia (ICD 9-CM code 427.1), including both sustained $(>30 \mathrm{~s})$ or non-sustained VT ( $\geq 3$ consecutive complexes), and ventricular fibrillation (ICD 9-CM code 427.4). Death was confirmed using data from Taiwan's National Death Registry. Follow-up was terminated in cases of death or if the patients lived beyond December 31, 2016.

\section{Statistical Analysis}

Normally distributed continuous variables are presented as mean values and standard deviations. Student's $t$-test was used to compare differences between two groups. Categorical variables were expressed as numbers and percentages and were compared using the Chi-square test. The incidence rates of mortality, CV death, and hospitalization were calculated as the number of cases per 1,000 person-years during followup. To minimize the influence of confounding factors on the clinical outcomes, propensity scores (PS) were utilized to match patients with mechanical valve and bioprosthetic valve replacements. A one-to-one matching of pairs was conducted using identical propensity scores with a 0.15 -caliper width for age, sex, hypertension, diabetes mellitus, chronic obstructive pulmonary disease, CHF, stroke, ESRD, and total site(s) of valve replacement.

The matched (conditional) Cox proportional-hazards regression model was utilized to compare the hazard ratios (HRs) with 95\% confidence intervals (CIs) of the outcomes. Potential confounders were adjusted using two models (Model 1, adjusted for age and sex; Model 2, adjusted for Model 1 factors 
plus total site(s) of valve replacement, hypertension, diabetes mellitus, CHF, coronary artery diseases, chronic obstructive pulmonary disease, prior stroke, and ESRD). The level of statistical significance was set at a two-tailed alpha level of $<0.05$. The analyses were performed using SAS software (version 9.4, SAS Institute, Cary, NC, USA).

\section{RESULTS}

\section{Selection and Characteristics of the Study Population}

In total, 19,528 patients who had undergone their first valve replacement were identified in the NHIRD. After excluding 883 patients, 18,645 were included in the original cohort, and 10,212 patients were selected after propensity score matching (PSM). After PSM, both the mechanical valve and bioprosthetic valve groups had an equal number of 5,106 patients (Figure 1).

Table 1 shows the baseline characteristics of the original and PSM cohorts. After PSM, more patients underwent bioprosthetic valve replacement for aortic, tricuspid, and pulmonary valves ( $P=0.04, P<0.001$, and $P<0.001$, respectively), while more patients underwent mechanical valve replacement for mitral valves $(P<0.001)$.

\section{Ventricular Tachyarrhythmias After Surgical Valve Replacement}

During a median follow-up period of 59.6 months (25-75\%, interquartile range $22.8-108.9), 288$ (2.8\%) patients with newly diagnosed VTa were identified, including 154 (1.5\%) and 134 (1.3\%) patients receiving surgical mechanical and bioprosthetic valve replacement, respectively $(P=0.23$, Figure 1$)$. The overall crude incidence rate of newly diagnosed VTa was 4.8/1,000 person-years. Among them, 4.8, and 4.7/1,000 person-years were identified in patients undergoing mechanical and bioprosthetic valve replacement, respectively (Table 2). Figure 2 shows the VTa-free survival curve after surgical valve replacement. After the early occurrence of VTa, the cumulative incidence of VTa persistently increased in the years following surgery.

After adjusting for the effects of age, sex, total site(s) of valve replacement, and underlying disease using a multivariate regression analysis, bioprosthetic valve replacement was significantly associated with a lower risk of hospitalization related to VTa $(P=0.009$, HR $0.63,95 \%$ CI $0.45-0.89)$ than those who underwent mechanical valve replacement during the 5-year follow-up (Table 3).

Patients with VTa after surgical valve replacement more likely undergo mitral and tricuspid valve replacement (59.0 vs. $50.7 \%$, $P=0.01 ; 7.3$ vs. $3.8 \%, P=0.003$, respectively), less likely undergo aortic valve replacement ( $49.3 \%$ vs. $57.2 \%, P=0.01)$, more likely undergo ICD implantation ( 11.1 vs. $0.1 \%, P<0.001)$, and have a history of CHF (32.6 vs. $26.2 \%, P=0.01$ ) (Table 4).

\section{Effect of Ventricular Tachyarrhythmias on Mortality and Hospitalization}

During long-term follow-up, the overall crude incidence rates of all-cause mortality and $\mathrm{CV}$ death after valve replacement were 91.0 and 47.7/1,000 person-years, respectively. Moreover, the crude incidence rates of all-cause mortality after valve replacement in patients with and without VTa were 128.8 and $89.9 / 1,000$ person-years, respectively, while the crude incidence rates of $\mathrm{CV}$ death after valve replacement in patients with and without VTa were 80.5 and 46.7/1,000 person-years, respectively (Table 2 ).

Compared with patients without VTa after surgical valve replacement using a multivariate regression analysis, patients with VTa were associated with a higher risk of $\mathrm{CV}$ death $(P<0.001$, HR 1.67, 95\% CI 1.41-1.96), stroke- $(P<0.001$, HR 1.66, 95\% CI 1.37-2.01), AF- $(P<0.001$, HR 2.80, 95\% CI 2.42-3.24), and CHF-related hospitalization $(P<0.001$, HR 2.61, 95\% CI 2.30-2.95) (Table 5). Moreover, a similar risk of all-cause mortality, CV death, stroke-, AF-, and CHFrelated hospitalization were observed between patients with VTa after surgical mechanical and bioprosthetic valve replacement (Supplementary Table 1).

In addition, lower all-cause mortality and CV death after VTa were observed in patients with ICD implantation after surgical valve replacement than in those without ICD implantation $(P<0.001$, HR $0.35,95 \%$ CI $0.20-0.59 ; P=0.002$, HR $0.34,95 \%$ CI 0.17-0.66, respectively; Table 6). However, the incidences of stroke-, AF-, and CHF-related hospitalization were comparable in patients experiencing VTa with and without ICD implantation after surgical valve replacement (Table 6).

\section{DISCUSSION}

\section{Major Findings}

This study has several notable findings. First, the crude incidence rate of newly diagnosed VTa after surgical valve replacement was 4.8/1,000 person-years during 5-year follow-up. In addition to the early occurrence of VTa after surgery, the cumulative incidence of VTa persistently increased in the following years. Second, compared with patients without VTa, patients with VTa after surgical valve replacement were associated with a higher risk of CV death, stroke-, AF-, and CHF-related hospitalization. Third, lower all-cause mortality and CV death after VTa were observed in patients undergoing ICD implantation after surgical valve replacement, reflecting the importance of evaluating the need for ICD implantation in these patients.

\section{Ventricular Tachyarrhythmias in Patients Undergoing Surgical Valve Replacement}

VTa frequently contributes to poor outcomes in patients with VHD, including reduced quality of life and increased risk of sudden cardiac arrest (1). Despite surgical replacement of VHD, several studies have reported that patients with VHD are still at risk for VTa even after valve replacement $(4,6-8)$. Konishi et al. reported that multiple valve replacement and larger heart size were associated with late occurrences of ventricular arrhythmias among long-term survivors who underwent aortic and/or mitral valve replacement (9). Similarly, in the present study, there was a trend toward more sites of surgical valve replacement in patients with VTa than those without (Table 4). On the other hand, patients with VTa after surgical valve replacement more 


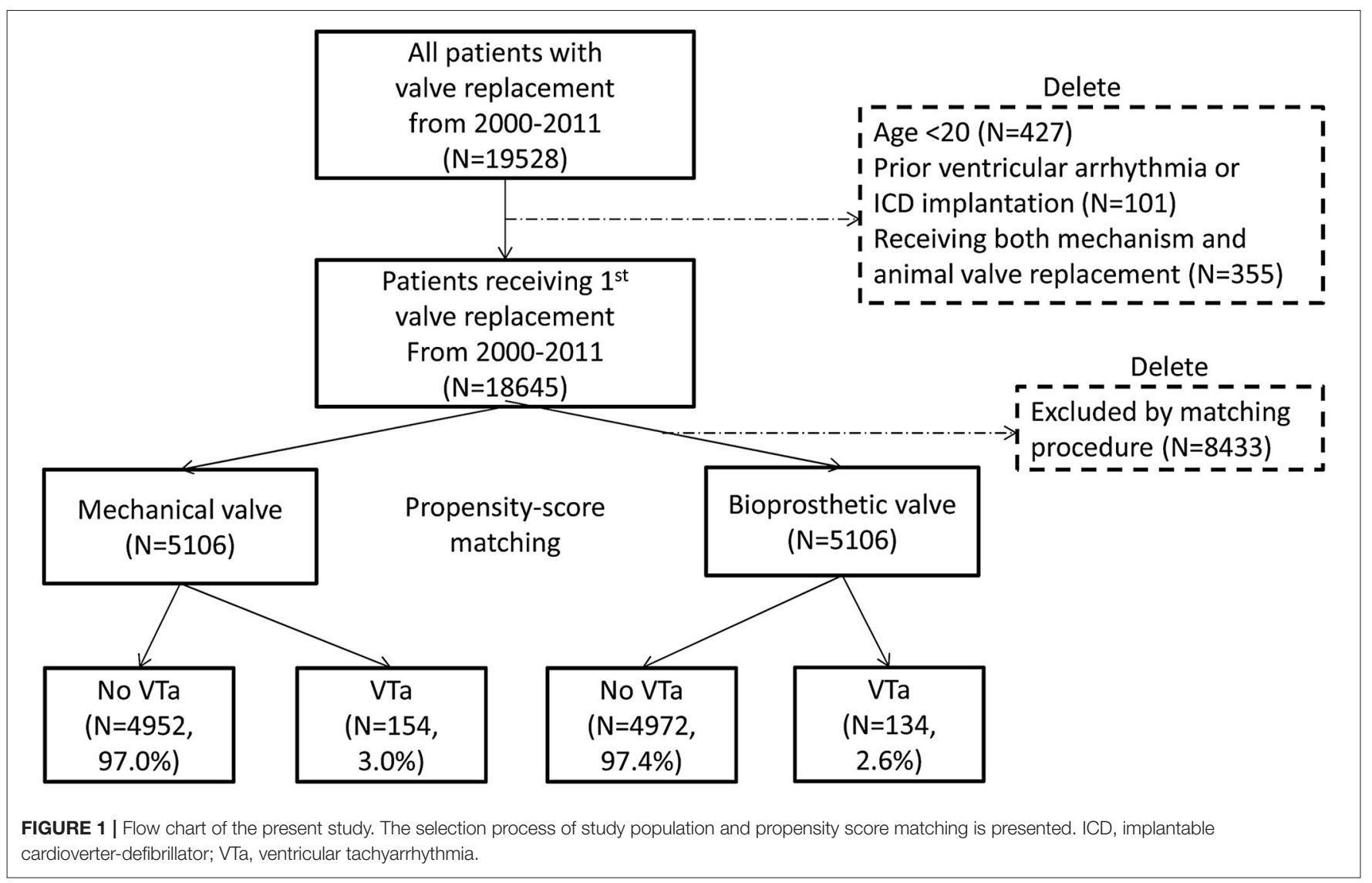

TABLE 1 | Baseline characteristics of the original cohort and propensity-score matched cohort.

\begin{tabular}{|c|c|c|c|c|c|c|}
\hline \multirow[t]{2}{*}{ Variables } & \multicolumn{3}{|c|}{ Original cohort (Total $N=18,645$ ) } & \multicolumn{3}{|c|}{ Propensity-score matched cohort (Total $N=10,212$ ) } \\
\hline & $\begin{array}{c}\text { Mechanical valve } \\
(N=13,537)\end{array}$ & $\begin{array}{c}\text { Bioprosthetic } \\
\text { valve }(N=5,108)\end{array}$ & $P$-value & $\begin{array}{c}\text { Mechanical } \\
\text { valve }(N=5,106)\end{array}$ & $\begin{array}{c}\text { Bioprosthetic } \\
\text { valve }(N=5,106)\end{array}$ & $P$-value \\
\hline Age & $58.4 \pm 14.6$ & $64.7 \pm 13.4$ & $<0.001$ & $64.7 \pm 13.5$ & $64.7 \pm 13.4$ & 0.98 \\
\hline Male sex & 7,947 (58.7\%) & $2,883(56.4 \%)$ & 0.005 & $2,857(56.0 \%)$ & $2,883(56.5 \%)$ & 0.60 \\
\hline \multicolumn{7}{|l|}{ Valve location } \\
\hline Aortic valve & 7,497 (55.4\%) & $2,966(58.1 \%)$ & 0.001 & $2,859(56.0 \%)$ & $2,964(58.0 \%)$ & 0.04 \\
\hline Mitral valve & $7,460(55.1 \%)$ & 2,500 (48.9\%) & $<0.001$ & 2,697 (52.8\%) & 2,500 (49.0\%) & $<0.001$ \\
\hline Tricuspid valve & 497 (3.7\%) & $239(4.7 \%)$ & 0.002 & 159 (3.1\%) & 239 (4.7\%) & $<0.001$ \\
\hline Pulmonary valve & $38(0.3 \%)$ & $49(1.0 \%)$ & $<0.001$ & $13(0.3 \%)$ & $49(1.0 \%)$ & $<0.001$ \\
\hline Total site(s) of valve replacement & $1.14 \pm 0.36$ & $1.13 \pm 0.35$ & 0.002 & $1.12 \pm 0.34$ & $1.13 \pm 0.35$ & 0.49 \\
\hline ICD implantation & $66(0.5 \%)$ & $22(0.4 \%)$ & 0.61 & $21(0.4 \%)$ & $22(0.4 \%)$ & 0.88 \\
\hline \multicolumn{7}{|l|}{ Underlying diseases } \\
\hline Diabetes mellitus (\%) & 597 (4.3\%) & $223(4.4 \%)$ & 0.79 & 200 (3.9\%) & $223(4.4 \%)$ & 0.25 \\
\hline Hypertension (\%) & $1,582(11.7 \%)$ & $596(11.7 \%)$ & 0.97 & $572(11.2 \%)$ & 596 (11.7\%) & 0.46 \\
\hline COPD (\%) & $218(1.6 \%)$ & $110(2.2 \%)$ & 0.01 & 95 (1.9\%) & $110(2.2 \%)$ & 0.29 \\
\hline Congestive heart failure (\%) & $3,028(22.4 \%)$ & $1,345(26.3 \%)$ & $<0.001$ & $1,350(26.4 \%)$ & $1,343(26.3 \%)$ & 0.88 \\
\hline Prior stroke (\%) & $468(3.6 \%)$ & 199 (3.9\%) & 0.35 & $183(3.6 \%)$ & 199 (3.9\%) & 0.40 \\
\hline Coronary artery disease (\%) & $472(3.5 \%)$ & $184(3.6 \%)$ & 0.70 & $166(3.3 \%)$ & $184(3.6 \%)$ & 0.33 \\
\hline End-stage renal disease (\%) & $1,091(8.1 \%)$ & $461(9.0 \%)$ & 0.03 & $470(9.2 \%)$ & $461(9.0 \%)$ & 0.76 \\
\hline
\end{tabular}

COPD, chronic obstructive pulmonary disease; $I C D$, implantable cardioverter-defibrillator. 
TABLE 2 | Incidence rates of subsequent events in various groups.

\begin{tabular}{|c|c|c|c|c|c|}
\hline Events & Groups & Total number & Total event number (\%) & Person-years (PY) & Incidence rate (per 1000 PYs) \\
\hline \multirow[t]{3}{*}{ VTa-related hospitalization } & Mechanical valve & 5,106 & 154 (3.0\%) & 32,229 & 4.8 \\
\hline & Bioprosthetic valve & 5,106 & $134(2.6 \%)$ & 28,299 & 4.7 \\
\hline & Total & 10,212 & $288(2.8 \%)$ & 60,528 & 4.8 \\
\hline \multirow[t]{3}{*}{ All-cause mortality } & Without VTa & 9,924 & $5,323(53.6 \%)$ & 59,194 & 89.9 \\
\hline & With VTa & 288 & $224(77.8 \%)$ & 1,740 & 128.8 \\
\hline & Total & 10,212 & $5,547(54.3 \%)$ & 60,934 & 91.0 \\
\hline \multirow[t]{3}{*}{ CV death } & Without VTa & 9,924 & 2,765 (27.9\%) & 59,194 & 46.7 \\
\hline & With VTa & 288 & $140(48.6 \%)$ & 1,740 & 80.5 \\
\hline & Total & 10,212 & 2,905 (28.4\%) & 60,934 & 47.7 \\
\hline
\end{tabular}

CV, cardiovascular; VTa, ventricular tachyarrhythmia.

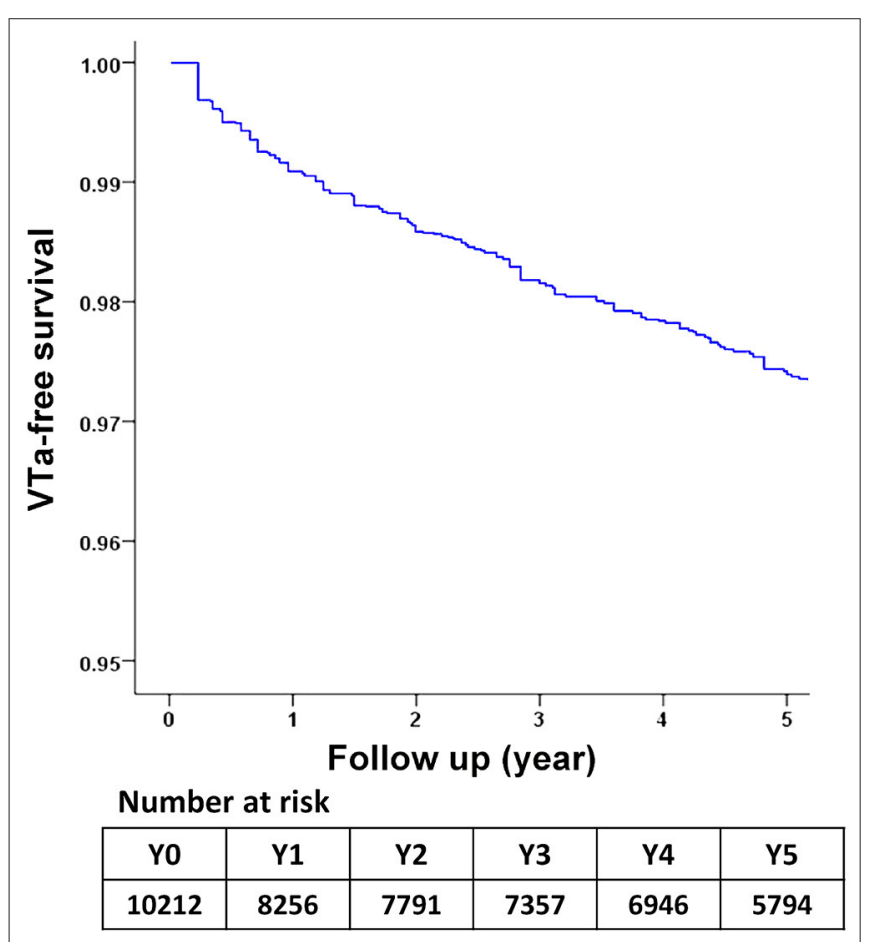

FIGURE 2 | The survival curve of freedom from VTa after surgical valve replacement during 5-year follow-up. VTa, ventricular tachyarrhythmia.

likely undergo mitral or tricuspid valve replacement and less likely undergo aortic valve replacement, implying that different sites of valvular replacement may contribute to ventricular arrhythmogenesis to a varying degree. However, the above findings and the associated mechanism require further validation and investigation.

Possible mechanisms of VTa in patients with VHD could be multifactorial. VT caused by bundle branch reentry (BBR) has been reported in patients with VHD, both before and after valve replacement (10-14). Narasimhan et al. demonstrated that BBR accounts for $30 \%$ of sustained monomorphic VT cases after valve surgery (15). It might be due to an impairment of the conduction system during surgery and, therefore, emerges as the most common type of VT in the early postoperative period. In addition, the postulated precipitating factors for ventricular arrhythmogenesis include invading calcification from the degenerated valve to the conduction system, mechanical irritation to the myocardium by the prosthetic valve, ischemia, heightened adrenergic state of the postoperative period, and surgical scar $(2,13,16-20)$.

The underlying diseased ventricular substrates in VHD may also increase the risk of VTa, which could be supported by the present finding of a higher incidence of CHF in those with VTa after surgical valve replacement. The correlation between VTa and left ventricular (LV) dysfunction in patients with VHD has also been reported in several studies (2127). Chronic pressure or volume overload of the myocardium could increase ventricular wall stress and structural remodeling, which subsequently enhances the formation of arrhythmogenic substrates even after surgical valve replacement, despite the fact that regression of ventricular arrhythmia in consonance with the improvement of LV systolic function has been reported after valve replacement $(28-30)$.

Michel et al. reported an increased frequency and worsening of ventricular arrhythmias early after aortic valve replacement (31). The early occurrence of VTa after valve replacement was also higher in the present study (Figure 2). However, the cumulative incidence of VTa persistently increased in subsequent years, which is consistent with the abovementioned mechanism of VTa. In addition to acute operative injury and mechanical irritation, chronic structural remodeling and diseased substrates also play roles in arrhythmogenesis and continue to increase the risk of VTa in the late postoperative stage.

\section{Influence of Ventricular Tachyarrhythmias and ICD Implantation After Surgical Valve Replacement}

Olafiranye et al. reported that non-sustained VT documented by 24-h ambulatory electrocardiography within 18 months after mitral valve surgery was associated with late postoperative cardiac mortality (32). Notably, sudden cardiac death is considered an important cause of cardiac mortality in these patients. Moreover, Hochreiter et al. demonstrated that most postoperative sudden deaths were observed in patients with 
TABLE 3 | Comparison of risk of ventricular tachyarrhythmias between bioprosthetic and mechanical valve replacement.

\begin{tabular}{|c|c|c|c|}
\hline \multirow[t]{2}{*}{ Groups } & \multirow[t]{2}{*}{ Risk factors } & \multicolumn{2}{|c|}{ Mechanical valve (ref) vs. bioprosthetic valve } \\
\hline & & Hazard ratio (95\% Cl) & $P$-value \\
\hline \multirow[t]{3}{*}{ VTa-related hospitalization } & Model 0 & $0.62(0.44-0.88)$ & 0.007 \\
\hline & Model 1 & $0.63(0.44-0.89)$ & 0.008 \\
\hline & Model 2 & $0.63(0.45-0.89)$ & 0.009 \\
\hline
\end{tabular}

Model 0: crude effect

Model 1: Model 0 plus age and sex.

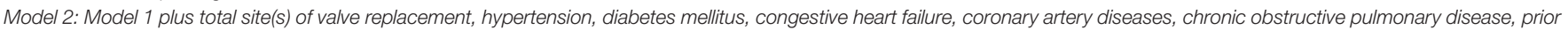
stroke, and end-stage renal disease.

VTa, ventricular tachyarrhythmia.

TABLE 4 | Baseline characteristics of patients with and without VTa after valve replacement.

\begin{tabular}{|c|c|c|c|}
\hline \multirow[t]{2}{*}{ Variables } & \multicolumn{3}{|c|}{ After propensity-score matching (Total $N=10,212$ ) } \\
\hline & Without VTa $(N=9,924)$ & With VTa $(N=288)$ & $P$-value \\
\hline Age & $64.7 \pm 13.5$ & $65.4 \pm 12.0$ & 0.33 \\
\hline Male sex & $5,588(56.3 \%)$ & $152(52.8 \%)$ & 0.23 \\
\hline \multicolumn{4}{|l|}{ Valve location } \\
\hline Aortic valve & $5,681(57.2 \%)$ & 142 (49.3\%) & 0.01 \\
\hline Mitral valve & $5,027(50.7 \%)$ & 170 (59.0\%) & 0.01 \\
\hline Tricuspid valve & $377(3.8 \%)$ & $21(7.3 \%)$ & 0.003 \\
\hline Pulmonary valve & $59(0.6 \%)$ & $3(1.0 \%)$ & 0.34 \\
\hline Total site(s) of valve replacement & $1.12 \pm 0.34$ & $1.17 \pm 0.38$ & 0.06 \\
\hline ICD implantation & $11(0.1 \%)$ & $32(11.1 \%)$ & $<0.001$ \\
\hline \multicolumn{4}{|l|}{ Underlying diseases } \\
\hline Diabetes mellitus (\%) & $410(4.1 \%)$ & $13(4.5 \%)$ & 0.75 \\
\hline Hypertension (\%) & $1,142(11.5 \%)$ & $26(9.0 \%)$ & 0.19 \\
\hline COPD (\%) & 199 (2.0\%) & $6(2.1 \%)$ & 0.93 \\
\hline Congestive heart failure (\%) & $2,599(26.2 \%)$ & $94(32.6 \%)$ & 0.01 \\
\hline Prior stroke (\%) & $372(3.7 \%)$ & $10(3.5 \%)$ & 0.81 \\
\hline Coronary artery disease (\%) & 342 (3.4\%) & $8(2.8 \%)$ & 0.54 \\
\hline End-stage renal disease (\%) & $912(9.2 \%)$ & $19(6.6 \%)$ & 0.13 \\
\hline
\end{tabular}

COPD, chronic obstructive pulmonary disease; ICD, implantable cardioverter-defibrillator; VTa, ventricular tachyarrhythmia.

documented non-sustained VT by routine Holter monitoring after surgery for chronic non-ischemic mitral regurgitation (33). Consistent with the above findings, we found that the presence of VTa was associated with various worse outcomes. Irrespective of the underlying $\mathrm{VHD}$, coexistent coronary artery disease, intraoperative injuries, and LV dysfunction may all play a role in the increased mortality and morbidity rate in patients with VTa after surgical valve replacement (15, 33).

Moreover, lower all-cause mortality and CV death after VTa were observed in patients with ICD implantation after surgical valve replacement than in those without, reflecting the potential role of ICD in the prevention of fatal ventricular arrhythmia in these high-risk patients. However, future studies are warranted to investigate potential surrogate markers for risk stratification of future fatal arrhythmias in patients who have not fulfilled the current recommendations for ICD implantation.

\section{Strengths and Limitations}

To the best of our knowledge, this is the first largescale study to investigate the clinical impact of VTa on various outcomes after surgical valve replacement using a nationwide population-based database. In addition, this study highlighted the importance of risk assessment for ICD implantation in patients with documented VTa after surgical valve replacement.

Nevertheless, this study has some limitations. First, given the retrospective design, some inevitable biases exist in this study. Second, despite PSM, some variables between the mechanical and bioprosthetic valve groups remained inconsistent. Third, diagnostic and procedural coding errors 
TABLE 5 | Comparison of various outcomes between patients with and without VTa after valve replacement.

\begin{tabular}{|c|c|c|c|}
\hline \multirow[t]{2}{*}{ Groups } & \multirow[t]{2}{*}{ Risk factors } & \multicolumn{2}{|c|}{ Without VTa (ref) vs. with VTa } \\
\hline & & Hazard ratio $(95 \% \mathrm{Cl})$ & $P$-value \\
\hline \multirow[t]{3}{*}{ All-cause mortality } & Model 0 & $1.43(1.28-1.58)$ & $<0.001$ \\
\hline & Model 1 & $1.39(1.22-1.59)$ & $<0.001$ \\
\hline & Model 2 & $1.01(0.89-1.15)$ & 0.84 \\
\hline \multirow[t]{3}{*}{ CV death } & Model 0 & $1.23(1.04-1.45)$ & 0.02 \\
\hline & Model 1 & $1.66(1.42-1.94)$ & $<0.001$ \\
\hline & Model 2 & $1.67(1.41-1.96)$ & $<0.001$ \\
\hline \multirow[t]{3}{*}{ Stroke-related hospitalization } & Model 0 & $1.70(1.40-2.06)$ & $<0.001$ \\
\hline & Model 1 & $1.67(1.37-2.02)$ & $<0.001$ \\
\hline & Model 2 & $1.66(1.37-2.01)$ & $<0.001$ \\
\hline \multirow[t]{3}{*}{ AF-related hospitalization } & Model 0 & $2.89(2.51-3.34)$ & $<0.001$ \\
\hline & Model 1 & $2.83(2.45-3.28)$ & $<0.001$ \\
\hline & Model 2 & $2.80(2.42-3.24)$ & $<0.001$ \\
\hline \multirow[t]{3}{*}{ CHF-related hospitalization } & Model 0 & $2.71(2.40-3.06)$ & $<0.001$ \\
\hline & Model 1 & $2.61(2.29-3.00)$ & $<0.001$ \\
\hline & Model 2 & $2.61(2.30-2.95)$ & $<0.001$ \\
\hline
\end{tabular}

Model 0: crude effect.

Model 1: Model 0 plus age and sex.

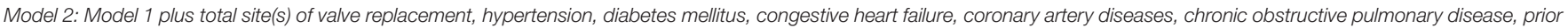
stroke, and end-stage renal disease.

$A F$, atrial fibrillation; CHF, congestive heart failure; CV, cardiovascular; VTa, ventricular tachyarrhythmia.

TABLE 6 | Comparison of various outcomes between patients experiencing VTa with and without ICD implantation.

\begin{tabular}{|c|c|c|c|}
\hline \multirow[t]{2}{*}{ Groups } & \multirow[t]{2}{*}{ Risk factors } & \multicolumn{2}{|c|}{ Without ICD (ref) vs. with ICD } \\
\hline & & Hazard ratio $(95 \% \mathrm{Cl})$ & $P$-value \\
\hline \multirow[t]{3}{*}{ All-cause mortality } & Model 0 & $0.32(0.19-0.56)$ & $<0.001$ \\
\hline & Model 1 & $0.34(0.20-0.57)$ & $<0.001$ \\
\hline & Model 2 & $0.35(0.20-0.59)$ & $<0.001$ \\
\hline \multirow[t]{3}{*}{ CV death } & Model 0 & $0.32(0.16-0.64)$ & 0.001 \\
\hline & Model 1 & $0.33(0.17-0.63)$ & 0.001 \\
\hline & Model 2 & $0.34(0.17-0.66)$ & 0.002 \\
\hline \multirow[t]{3}{*}{ Stroke-related hospitalization } & Model 0 & $0.64(0.32-1.25)$ & 0.19 \\
\hline & Model 1 & $0.65(0.33-1.26)$ & 0.20 \\
\hline & Model 2 & $0.71(0.36-1.40)$ & 0.32 \\
\hline \multirow[t]{3}{*}{ AF-related hospitalization } & Model 0 & $0.96(0.58-1.59)$ & 0.86 \\
\hline & Model 1 & $1.09(0.65-1.81)$ & 0.74 \\
\hline & Model 2 & $0.99(0.59-1.66)$ & 0.98 \\
\hline \multirow[t]{3}{*}{ CHF-related hospitalization } & Model 0 & $1.04(0.67-1.59)$ & 0.88 \\
\hline & Model 1 & 1.07 (0.70-1.64) & 0.74 \\
\hline & Model 2 & $1.24(0.83-1.88)$ & 0.30 \\
\hline
\end{tabular}

Model 0: crude effect.

Model 1: Model 0 plus age and sex.

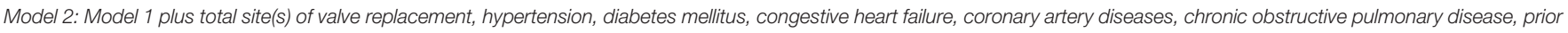
stroke, and end-stage renal disease.

AF, atrial fibrillation; CHF, congestive heart failure; CV, cardiovascular; ICD, implantable cardioverter-defibrillator; VTa, ventricular tachyarrhythmia.

might exist. Fourth, some important parameters, such as left ventricular ejection fraction during peri-procedural period, extent of $\mathrm{CAD}$, or detailed characteristics of VTa, cannot be extracted from the National Health Insurance Research
Database. In addition, some detailed information was not available in the present study, including the pre-procedural between-group distribution of $\mathrm{AF}$, or concomitant $\mathrm{CABG}$ in valve replacement. Future prospective researches will be warranted 
to clarify the above concerns. Finally, this study does not explore surrogate markers for future risk stratification and identify candidates for ICD implantation in addition to current recommendations. Future investigations are needed to elucidate the high-risk population in patients with VTa after surgical valve replacement.

\section{CONCLUSIONS}

The crude incidence rate of VTa after surgical valve replacement was $4.8 / 1,000$ person-years, which is attributed to both early and late postoperative VTa. The presence of VTa after surgical valve replacement significantly led to higher CV death and hospitalization rates due to various causes, while ICD implantation reduced the mortality rate in these patients.

\section{DATA AVAILABILITY STATEMENT}

The raw data supporting the conclusions of this article will be made available by the authors, without undue reservation.

\section{REFERENCES}

1. Pedersen CT, Kay GN, Kalman J, Borggrefe M, Della-Bella P, Dickfeld T, et al. EHRA/HRS/APHRS expert consensus on ventricular arrhythmias. Heart Rhythm. (2014) 11:e166-96. doi: 10.1016/j.hrthm.2014.07.024

2. Janse MJ. Possible mechanisms of arrhythmias in valvular heart disease. Acta Cardiol. (1992) 47:93-8.

3. Bloomfield P, Wheatley DJ, Prescott RJ, Miller HC. Twelve-year comparison of a Bjork-Shiley mechanical heart valve with porcine bioprostheses. $N$ Engl J Med. (1991) 324:573-9. doi: 10.1056/NEJM199102283240901

4. Blackstone EH, Kirklin JW. Death and other time-related events after valve replacement. Circulation. (1985) 72:753-67. doi: 10.1161/01.CIR.72.4.753

5. Hsu CC HC, Chen HC. 2015 Annual Report of Kidney Disease in Taiwan. Taipei: National Health Research Institutes (2016).

6. Kostis JB, Tupper B, Moreyra AE, Hosler M, Cosgrove N, Terregino C. Aortic valve replacement in patients with aortic stenosis. Effect on cardiac arrhythmias. Chest. (1984) 85:211-4. doi: 10.1378/chest.8 5.2 .211

7. Lewalter T, Jung W, Preusse CJ, Lickfett L, Wolpert C, Yang A, et al. Radiofrequency catheter ablation of an incessant ventricular tachycardia following valve surgery. Pacing Clin Electrophysiol. (2002) 25:105-8. doi: 10.1046/j.1460-9592.2002.t01-1-00105.x

8. Harrild DM, Berul CI, Cecchin F, Geva T, Gauvreau K, Pigula F, et al. Pulmonary valve replacement in tetralogy of Fallot: impact on survival and ventricular tachycardia. Circulation. (2009) 119:445-51. doi: 10.1161/CIRCULATIONAHA.108.775221

9. Konishi Y, Matsuda K, Nishiwaki N, Shimada I, Kitao Y, Ban T, et al. Ventricular arrhythmias late after aortic and/or mitral valve replacement. Jpn Circ J. (1985) 49:576-83. doi: 10.1253/jcj.49.576

10. Touboul P, Kirkorian G, Atallah G, Moleur P. Bundle branch reentry: a possible mechanism of ventricular tachycardia. Circulation. (1983) 67:67480. doi: 10.1161/01.CIR.67.3.674

11. Singh G, Lahiri MK, Khan A, Schuger CD. Bundle branch reentrant ventricular tachycardia after transcatheter aortic valve replacement. HeartRhythm Case Rep. (2017) 3:177-85. doi: 10.1016/j.hrcr.2016. 12.005

12. de la Rosa Riestra A, Rubio Caballero JA, Freites Estevez A, Alonso Bello J, Botas Rodriguez J. Bundle-branch reentry ventricular tachycardia after transcatheter aortic valve replacement. Indian Pacing Electrophysiol J. (2015) 15:251-4. doi: 10.1016/j.ipej.2016.02.004

\section{AUTHOR CONTRIBUTIONS}

All authors listed have made a substantial, direct and intellectual contribution to the work, and approved it for publication.

\section{FUNDING}

This work was supported by the Center for Dynamical Biomarkers and Translational Medicine, Ministry of Science and Technology (Grant Nos. MOST 109-2314-B-075-074-MY3 and MOST 106-2314-B-075-006-MY3), Research Foundation of Cardiovascular Medicine, Szu-Yuan Research Foundation of Internal Medicine (Grant Nos. 109047 and 109002), and Taipei Veterans General Hospital (Grant Nos. V107B-014, V108C-107, and V109C-113).

\section{SUPPLEMENTARY MATERIAL}

The Supplementary Material for this article can be found online at: https://www.frontiersin.org/articles/10.3389/fcvm. 2021.676897/full\#supplementary-material

13. Fuller M, Reithmann C, Becker A, Remp T, Kment A, Steinbeck G. Bundle branch reentrant tachycardia in a patient with a calcified bicuspid aortic valve and normal ventricular function. Clin Res Cardiol. (2006) 95:16873. doi: 10.1007/s00392-006-0343-5

14. Singh B, Sudan D, Kaul U. Radiofrequency ablation for bundle branch reentrant tachycardia in a patient with aortic valve replacement and left ventricular dysfunction. Indian Heart J. (1998) 50:551-3.

15. Narasimhan C, Jazayeri MR, Sra J, Dhala A, Deshpande S, Biehl M, et al. Ventricular tachycardia in valvular heart disease: facilitation of sustained bundle-branch reentry by valve surgery. Circulation. (1997) 96:430713. doi: 10.1161/01.CIR.96.12.4307

16. Eckart RE, Hruczkowski TW, Tedrow UB, Koplan BA, Epstein LM, Stevenson WG. Sustained ventricular tachycardia associated with corrective valve surgery. Circulation. (2007) 116:200511. doi: 10.1161/CIRCULATIONAHA.107.703157

17. Zaker-Shahrak R, Altmann D, Sommer P, Gaspar T, Schönbauer R, Arya A. Sustained monomorphic left ventricular outflow tract tachycardia early after aortic valve replacement. Cardiol J. (2012) 19:320-2. doi: 10.5603/CJ.2012.0057

18. Yamada T, McElderry HT, Doppalapudi H, Kay GN. Successful radiofrequency catheter ablation of ventricular tachycardia originating from underneath the mechanical prosthetic aortic valve. Pacing Clin Electrophysiol. (2008) 31:61820. doi: 10.1111/j.1540-8159.2008.01052.x

19. Tohoku S, Hiroshima K, Kuramitsu S, Nagashima M, Fukunaga M, An Y, et al. Radiofrequency ablation of ventricular premature contraction originating from a native coronary cusp after transcatheter aortic valve replacement. $J$ Arrhythm. (2017) 33:630-2. doi: 10.1016/j.joa.2017.08.006

20. Dvir D, Haim M, Kornowski R. Left-ventricular outflow tract ventricular-tachycardia event following CoreValve transcatheter aortic-valve implantation. Catheter Cardiovasc Interv. (2012) 79:331-3. doi: 10.1002/ccd.23183

21. Viguier E, Delahaye JP, de Gevigney G, Garé JP, Delahaye F, Michel PL, et al. [Pre- and postoperative ventricular arrhythmia in mitral valve insufficiency]. Arch Mal Coeur Vaiss. (1994) 87:439-44.

22. Shigemitsu O, Hadama T, Takasaki H, Uchida Y, Shirabe J, Ito M. Analysis of perioperative ventricular arrhythmias in valvular heart diseases by Holter ECG recording. Jpn Circ J. (1991) 55:951-61. doi: 10.1253/jcj.55.951

23. Meinertz T, Kasper W, Bödecker W, Hofmann T, Zehender M, Geibel A, et al. [Relation between hemodynamics and ventricular arrhythmia in patients with heart valve diseases]. Z Kardiol. (1987) 76:421-7. 
24. von Olshausen K, Treese N, Schwarz F, Kübler W, Meyer J. [Ventricular arrhythmias in mitral valve disease: incidence, severity and relations to hemodynamic parameters]. Z Kardiol. (1986) 75:196-201.

25. Martínez-Useros C, Tornos P, Montoyo J, Permanyer Miralda G, Alijarde M, García del Castillo H, et al. Ventricular arrhythmias in aortic valve disease: a further marker of impaired left ventricular function. Int J Cardiol. (1992) 34:49-56. doi: 10.1016/0167-5273(92)90081-D

26. Santinga JT, Kirsh MM, Brady TJ, Thrall J, Pitt B. Left ventricular function in patients with ventricular arrhythmias and aortic valve disease. Ann Thorac Surg. (1983) 35:152-5. doi: 10.1016/S0003-4975(10)61452-X

27. von Olshausen K, Schwarz F, Hennig E, Krämer B, Kübler W. [Ventricular arrhythmias in patients with aortic valve disease (author's transl)]. Z Kardiol. (1981) 70:895-901.

28. von Olshausen K, Amann E, Hofmann M, Schwarz F, Mehmel HC, Kübler W. Ventricular arrhythmias before and late after aortic valve replacement. Am J Cardiol. (1984) 54:142-6. doi: 10.1016/0002-9149(84)90319-9

29. Tempio D, Pruiti GP, Conti S, Romano SA, Tavano E, Capodanno $\mathrm{D}$, et al. Ventricular arrhythmias in aortic valve stenosis before and after transcatheter aortic valve implantation. Europace. (2015) 17:113640. doi: 10.1093/europace/euu362

30. Calle-Muller C, Rabbani B, Khan A, Guerrero M. Recurrent ventricular tachycardia from severe aortic stenosis improves after percutaneous aortic balloon valvuloplasty and transcatheter aortic valve replacement. J Cardiol Cases. (2014) 9:236-8. doi: 10.1016/j.jccase.2014.03.005
31. Michel PL, Mandagout O, Vahanian A, Cormier B, Iung B, Luxereau P, et al. Ventricular arrhythmias in aortic valve disease before and after aortic valve replacement. Acta Cardiol. (1992) 47:145-56.

32. Olafiranye O, Hochreiter CA, Borer JS, Supino PG, Herrold EM, Budzikowski AS, et al. Nonischemic mitral regurgitation: prognostic value of non-sustained ventricular tachycardia after mitral valve surgery. Cardiology. (2013) 124:10815. doi: 10.1159/000347085

33. Hochreiter C, Borer JS, Yin A, Supino PG, Herrold EM, Krieger $\mathrm{K}$, et al. Ventricular arrhythmias in mitral regurgitation: frequency, clinical and prognostic importance, management before and after mitral valve surgery. Adv Cardiol. (2004) 41:108-11. doi: 10.1159/0000 79789

Conflict of Interest: The authors declare that the research was conducted in the absence of any commercial or financial relationships that could be construed as a potential conflict of interest.

Copyright (c) 2021 Li, Chen, Chien, Lin, Kuo and Chung. This is an open-access article distributed under the terms of the Creative Commons Attribution License (CC $B Y)$. The use, distribution or reproduction in other forums is permitted, provided the original author(s) and the copyright owner(s) are credited and that the original publication in this journal is cited, in accordance with accepted academic practice. No use, distribution or reproduction is permitted which does not comply with these terms. 